\title{
The method of dynamic operating modes investigation in electrohydraulic drive systems with improved accuracy of positioning executive elements
}

\author{
Valeriy V. Grechikhin*, Galina A. Galka, Anatoliy I. Ozerskiy, and Mikhail E. Shoshiashvili \\ Platov South-Russian State Polytechnic University (NPI), Department of Applied Mathematics, \\ 346428 Novocherkassk, Russia
}

\begin{abstract}
The article describes the method of dynamic operating modes investigation in electrohydraulic drive systems with improved accuracy of positioning output element. The method is the evolution of the fundamental positions of the mechanics of continuous media with moving boundaries as applied to the research of non-stationary processes accompanying the operation of hydraulic drive systems with piston hydraulic machines. The method is based on generalized modeling (technical, physical, mathematical and computer), takes into account the peculiarities of mutual influence of electric and hydraulic machines during their joint work as part of the electrohydraulic drive, which raises the level and adequacy of actuators simulation, as well as the reliability of the assessment of their technical condition. The method extends the field of research, improves the accuracy of the calculation of the positioning of the executive elements, taking into account the different dynamic modes of the drives under study.
\end{abstract}

\section{Introduction}

Electrohydraulic drive systems (EHDS) with volumetric hydraulic machines (VHM) are effective power systems with high speed and positioning accuracy of output power elements [1]. These systems are widely used in machine tools, robotics and mechatronics. The calculation of the dynamic modes of operation of such systems is one of the most urgent and complex tasks of modern mechanical engineering.

Despite the fact that a significant amount of work is devoted to the creation and improvement of methods for calculating these systems, many tasks remain unresolved. This is because the methods of assessing their technical condition, which exist at the present time, are based on calculations with the help of a computer and do not take into account the complexity of the processes of joint operation of electric motors with hydraulic drive machines, the peculiarities of their interaction among themselves, since they are not considered as a single electrohydromechanical systems (EHMS). This narrows the field of

\footnotetext{
* Corresponding author: vgrech@mail.ru
} 
research of dynamic modes of operation of systems, reduces accuracy and reliability of calculations, and also the quality and adequacy of constructed computer models in relation to real processes and objects [2-6].

In this regard, the solution to the problem of creating methods of dynamic operating modes investigation in EHDS with improved accuracy of positioning executive elements is actual.

The modern level of knowledge of the features of the dynamic modes of operation of the systems investigated in this article shows that the processes accompanying their operation under real conditions are much more complicated than those inherent in conventional EHDS that do not possess high accuracy of positioning [1]. This is due to the high shock starting currents and torque of the electric motor, as well as to the complexity of the physical phenomena accompanying the operation of hydraulic drive systems with multi-piston VHM, which are usually used in high-precision power drives [1]. The complexity of these phenomena is determined by the high-speed processes of filling and emptying the working cavities of hydraulic cylinders of volumetric drive machines, which constantly accompany their work [1]. Such processes are typical for the operation of valves, dampers and other controls and regulation of the drive.

Efficiency and position accuracy of the drive is determined by the accuracy of providing a specified (calculated) change in the coordinates of its actuators, as well as the accuracy of providing design fluid pressure on the surfaces of the actuators. Therefore, it is necessary to calculate the coordinates of the boundaries of liquid media with the surfaces of the mobile power elements of the drive in the computer simulation of the systems investigated in the article, namely, with the surfaces of the pistons of power cylinders, pistons of volumetric hydraulic pumps and hydraulic motors. All this determines the quality of process modeling and allows increasing the positional accuracy of EHDS with volumetric hydraulic machines.

In the general case, the problems of investigating the processes of motion of liquid media with moving interfaces between media such as "liquid-gas" or "liquid-solid" ("liquidpiston") belong to problems of hydromechanics with contact discontinuities of media [7].

The necessity to determine the laws of motion of moving interfaces between media (boundaries of discontinuities) is one of the main tasks in investigating the features of the processes considered in the article. However, the area for setting and solving hydromechanical problems with moving media boundaries for the systems investigated here is limited by the Euler principle, traditionally used in machine-building hydraulics. Researches show that, in addition to the Euler principle, in this case, it is also necessary to apply the Lagrange principle, which in physical and mathematical modeling is more complex, but more general, since it allows one to set and solve hydromechanical problems with moving boundaries of working fluids [7].

The use of the Lagrange principle makes it possible to develop the fundamental principles of the mechanics of liquid media with variable boundaries, applying them to the dynamic problems of hydraulic systems of high-precision drives with moving boundaries of liquid working bodies. This greatly expands the field of research, as well as the formulation and solution of problems of calculating the dynamic operating modes of systems modeled in this article. The accuracy and reliability of calculations is increased, as well as the adequacy of systems of the modeling in relation to real objects and processes that accompany their work $[5,6]$.

\section{Theoretical basis of the calculation method}

The theoretical foundations of the method of calculation described in the article are general integral relations expressing the basic laws of the mechanics of fluid media with moving 
boundaries, namely, the laws of changing the mass $m(t)$ of the media, its amount of motion $\bar{K}(t)$, kinetic energy $E(t)$ and moment $\bar{L}(t)$ amount of motion [7]. They are presented here in the form of equations:

$$
\begin{gathered}
\frac{d m}{d t}=\frac{d}{d t} \int_{V(t)} \rho d V=\int_{V(t)} \frac{\partial \rho}{\partial t} d V+\int_{\sigma(t)} \rho \mathrm{v}_{n} d \sigma=\sum_{(i, j)} \dot{m}_{i, j}, \\
\frac{d E}{d t}=\frac{d}{d t} \int_{V(t)} \rho \frac{1}{2} v^{2} d V=\int_{V(t)} \frac{\partial}{\partial t}\left[\rho \frac{1}{2} v^{2}\right] d V+\int_{\sigma(t)} \rho \frac{1}{2} v^{2} v_{n} d \sigma= \\
=\int_{V(t)} \rho \cdot \bar{f} \cdot \bar{v} d V+\int_{\sigma(t)} \bar{p} \cdot \overline{\mathrm{v}}_{n} d \sigma+\sum_{i, j} \dot{E}_{i, j}, \\
\frac{d \bar{K}}{d t}=\frac{d}{d t} \int_{V(t)} \rho \overline{\mathrm{v}} d V=\int_{V(t)} \frac{\partial}{\partial t}(\rho \overline{\mathrm{v}}) d V+\int_{\sigma(t)}(\rho \overline{\mathrm{v}}) \mathrm{v}_{n} \cdot d \sigma= \\
=\int_{V(t)} \bar{f} \rho d V+\int_{\sigma(t)} \bar{p}_{n} d \sigma+\sum_{(i, j)} \overline{\dot{K}}_{i, j} \\
\frac{d \bar{L}}{d t}=\frac{d}{d t} \int_{V(t)}(\bar{r} \times \rho \overline{\mathrm{v}}) d V=\int_{V(t)} \frac{\partial}{\partial t}(\bar{r} \times \rho \bar{v}) d V+\int_{\sigma(t)}(\bar{r} \times \rho \bar{v}) \mathrm{v}_{n} d \sigma= \\
=\int_{V(t)}(\bar{r} \times \rho \bar{f}) d V+\int_{\sigma(t)}\left(\bar{r} \times \bar{p}_{n}\right) d \sigma+\sum_{(i, j)} \overline{\dot{L}}_{i, j} .
\end{gathered}
$$

Here $\bar{v}-$ is the absolute velocity vector of a given particle of the medium; $\rho-$ is the density of the medium; $\bar{f}$ and $\bar{p}$ - is the vectors of the strengths of the mass and surface forces acting on a particle of the medium and its surface respectively; $p_{n}$ and $p_{\tau}-$ is normal and tangential to the surface components of the vector $\bar{p}$ respectively; $\sum_{(i, j)} \dot{m}_{i, j}, \sum_{(i, j)} \dot{E}_{i, j}-$ is the sum of the capacities of additional sources and sinks of mass and mechanical energy of the fluid, respectively, located inside the moving liquid medium; $\sum_{(i, j)} \overline{\dot{K}}_{i, j}, \sum_{(i, j)} \overline{\dot{L}}_{i, j}-$ is the sum of the capacities of additional sources and sinks of the amount of motion and the moment amount of motion of the liquid, respectively, located inside the liquid; $\bar{r}$ - radius vector drawn at a given point of the medium from the center of its rotation.

Elements of the theory are developed in the paper [7], the method for calculating and computer simulation of nonstationary processes of motion of liquid and gas-liquid media with moving media boundaries in the channels of complex geometric shapes of these hydraulic machines and highways are created. Here are considered the one-dimensional unsteady motion of a fluid medium in a channel whose cross-sectional area $\sigma(s)$ is a given function of the curvilinear coordinate $s$, measured from some fixed point along the channel axis.

It can be shown that equations (1) - (4) can be transformed for one-dimensional motions of fluid media moving in the channels of hydraulic machines and lines and bounded by moving boundaries with the coordinates $s_{1}(t)$ and $s_{2}(t)$, by differentiating with respect to time the quantities: mass $m(t)$ of the fluid, its kinetic energy $E(t)$, amount of 
motion $\bar{K}(t)$ and moment $\bar{L}(t)$ amount of motion, expressed, respectively, in the form of integrals with variable boundaries:

$$
\begin{array}{cc}
m(t)=\int_{S_{1}(t)}^{S_{1}(t)} \rho(s, t) \sigma(s) d s, & E(t)=\frac{1}{2} \int_{S_{1}(t)}^{S_{1}(t)} \rho(s, t) \mathrm{v}^{2}(s, t) \sigma(s) d s, \\
\bar{K}(t)=\int_{S_{1}(t)}^{S_{1}(t)} \rho(s, t) \overline{\mathrm{v}}(s, t) \sigma(s) d s, & \bar{L}(t)=\int_{S_{1}(t)}^{S_{1}(t)}[\bar{r}(s, t) \times \rho(s, t) \overline{\mathrm{v}}(s, t)] \sigma(s) d s .
\end{array}
$$

The equation for calculating the one-dimensional motion of a viscous incompressible fluid with moving boundaries in the channels of hydraulic lines can be represented in form

$$
a(x, t) \ddot{x}=b(x, t) \dot{x}^{2}+c(x, t)
$$

where $x=x(t)$ is the coordinate of the front or rear boundary of the medium moving in the channel.

Equation (5) is the generalized Bernoulli equation for the case of one-dimensional motion of fluid media with moving boundaries. It is similar to the equation of motion of a body with a variable mass.

Thus, the problem of calculating the processes of one-dimensional motion of an incompressible fluid medium with moving boundaries in a channel reduces to the solution of an ordinary second-order nonlinear differential equation with respect to the coordinate of one of the moving boundaries of the media. This is the main difference between the method described in this article and the method traditionally used in hydraulics, based on Euler's approach, in which a fluid-filled section of a channel with fixed boundaries is considered. Investigations in the method based on Euler's approach are known to reduce to the solution of an ordinary differential first-order nonlinear equation with respect to the volume flow $Q$ of a liquid:

$$
\dot{Q}=b(t) Q^{2}+c(t)
$$

The calculation of the dynamic modes of the electro-hydraulic system with moving boundary liquid medium in the hydraulic channels and the working cavities of blade machines in this method is reduced to the solution of a system of ordinary nonlinear differential equations of the form $[7,8]$ :

$$
\begin{gathered}
A_{1}(x) \ddot{x}+B_{1}(x) \ddot{\varphi}+C_{1}(x) \dot{x}^{2}+D_{1}(x) \dot{\varphi}+E_{1}(x) \dot{x} \dot{\varphi}=\Delta P-\Delta P_{L} \\
A_{2}(x) \ddot{x}+B_{2}(x) \ddot{\varphi}+C_{2}(x) \dot{x}^{2}+D_{2}(x) \dot{\varphi}+E_{2}(x) \dot{x} \dot{\varphi}=M_{P} \\
\sum_{j=\alpha}^{q} L_{i, j} \frac{d i_{j}}{d t}+\dot{\varphi} \sum_{j=\alpha}^{q} \frac{d L_{i, j}}{d \varphi} i_{j}=u_{i}-R_{i} i_{i} \\
\frac{1}{2} \sum_{i=\alpha}^{q} i_{i} \sum_{j=\alpha}^{q} \frac{d L_{i, j}}{d \varphi} i_{j}=M_{E} ; i, j=\alpha, \beta, d, q \\
J_{\Sigma} \ddot{\varphi}=M_{E}-M_{F}-M_{P}
\end{gathered}
$$

where $x=x(t)$ - is coordinate of any moving boundary of the medium, for example $x=s_{2}(t) ; \varphi(t)$ - is angle of rotation of the machine shaft; $\Delta P(x, t)$ - is the difference 
between the mean values of the fluid pressure on the surfaces of moving boundaries; $\Delta P_{L}-$ is loss of pressure due to dissipation of mechanical energy of the liquid medium flow; $M_{P}(x, t)$ - is torque on the pump drive shaft; $M_{E}$ - is rotating electromagnetic moment produced by an electric motor (EM); $M_{F}$ - is moment of friction forces preventing rotation of the rotor shaft of EM; $J_{\Sigma}$ - is this is the sum moment of inertia, taking into account the inertia of the rotating parts, both the rotor of the electric motor, and the inertia of the rotating parts of the pump, leding to the shaft of its rotor.

The system of these equations includes unknown functions: the coordinate $x=x(t)$ of any moving boundary of the liquid medium and the angle $\varphi(t)$ of rotation of the shaft of the hydraulic machine. The unknowns are also the projections $i_{\alpha}, i_{\beta}, i_{d}, i_{q}$ of the electric currents in the stator and rotor windings (indices $\alpha$ and $\beta, d$ and $q$, respectively) on the axis of the rectangular coordinate systems rigidly connected with the stator ( $\alpha$ and $\beta$ ) and the rotor $(d$ and $q)$, respectively.

In these equations: $R_{i}$ and $u_{i}(i=\alpha, \beta, d, q)$ respectively, the electrical resistance of the stator and rotor windings and the electrical stresses at the ends of these windings; $L_{i, j}-$ corresponding to the lower indices $(i, j=\alpha, \beta, d, q)$ of the inductance of the stator windings and the rotor of ED as a generalized two-phase electric machine [8]. The coefficients of equations (6) and (7) are determined by the geometry of the channels of the working wheels of blade machines.

It should be noted that the methods for calculating the dynamic operating modes of electrohydraulic systems with paddle and volumetric hydraulic machines are significantly different. This is due to the different operating principles of these machines [1]. As is known, blade machines are "flowing": here the entrance to the machine is constantly and freely communicating with the exit through the channels between the blades. Volumetric machines, on the contrary, are "hermetic": here the entrance to the car is constantly and hermetically separated from the exit. Therefore, filling the channel with a volume pump (in contrast to a channel with a blade pump) is possible only when the shaft of its drive rotates. In this regard, the functions $x(t)$ and $\varphi(t)$ are no longer independent here, as in the case of blade pumps. The position $x(t)$ of the moving fluid boundary in the case of using volumetric machines with an electric motor is directly dependent on the angle $\varphi(t)$ of rotation of the motor shaft. Therefore, the calculation of dynamic operating modes of EHDS with volumetric hydraulic machines is limited to the solution of equations (8) - (10). In this case, the velocities and accelerations of the fluid boundaries of the liquid, i.e. $\dot{x}(t)$ and $\ddot{x}(t)$, are determined by the functions $\dot{\varphi}(t)$ and $\ddot{\varphi}(t)$. Unknown functions $x(t), \varphi(t)$ and $i_{i}$ are defined here in the process of calculations and mathematical modeling of a single EHDS.

\section{Conclusions}

The method of dynamic operating modes investigation in electrohydraulic drive systems with improved accuracy of positioning executive elements is developed. As the results of technical and computer experiments show, the method created allows with an acceptable accuracy $(15-20 \%)$ to calculate the dynamic operating modes of electrohydraulic drive systems with moving boundaries of working fluids. The application of this method can expand the scope of research of dynamic operating modes of the power electrohydraulic drive on the basis of computer simulation and virtual computer tests. 
The study results are obtained with the support of the project \#2.7193.2017/8.9 "Development of scientific bases of design, identification and diagnosis systems for highly accurate positioning with application of the methodology of inverse problems of electrical engineering", carried out within the framework of the base part of State job.

\section{References}

1. V.N. Prokofiev, Machine-building hydraulic drive (Mashinostroenie, Moscow, 1978)

2. A.I Ozerskiy, Yu.I. Babenkov, M.E Shoshiashvili, University News. North-Caucasian Region. Technical Sciences Series, 6, 55 (2008)

3. A.I Ozerskiy, Vestnik of Don State Technical University, 10, 6 (49), 914 (2010)

4. A.I Ozerskiy, M.E Shoshiashvili, University News. North-Caucasian Region. Technical Sciences Series, 1, 52 (2014)

5. A.I. Ozerskiy University News. North-Caucasian Region. Technical Sciences Series, 5, 37 (2013)

6. A.I. Ozerskiy, M.E. Shoshiashvili, Proc. of the Computer Modeling and Simulation, (2014)

7. A.I. Ozerskiy, D.A. Polukhin, V.S. Sizonov, Proceedings of the Academy of Sciences of the USSR. Energy and Transport, 2, 143 (1979)

8. V.I. Klyuchev, Electric drive theory (Energoatomizdat, Moscow, 1985) 